\title{
Surgical resection of recurrent inferior vena cava tumor following radical nephrectomy for renal cell carcinoma: A case report
}

\author{
GUANGYUAN LI ${ }^{1,2}$, ZHIQIANG ZHANG ${ }^{1}$, DONGDONG XIE ${ }^{1}$, NAN YE ${ }^{2}$ and DEXIN YU \\ ${ }^{1}$ Department of Urology, Second Affiliated Hospital of Anhui Medical University; ${ }^{2}$ Department of Urology, \\ Fourth Affiliated Hospital of Anhui Medical University, Hefei, Anhui 230022, P.R. China
}

Received August 6, 2014; Accepted April 14, 2015

DOI 10.3892/ol.2015.3187

\begin{abstract}
Late recurrence is a known characteristic of the biological behavior of renal cell carcinoma (RCC) following radical nephrectomy. However, the development of recurrent inferior vena cava (IVC) tumors following radical nephrectomy for RCC is a rare event, and surgical resection of recurrent IVC tumors is a challenge for urologists. The present study reports the case of a patient with a local recurrent tumor in the IVC following a right radical nephrectomy 4 years previously for RCC. The patient was referred to the Department of Urology, First Affiliated Hospital of Anhui Medical University, due to bilateral lower extremity edema, and magnetic resonance imaging showed an intraluminal tumor thrombus in the IVC. Therefore, a thrombectomy and partial IVC resection with defect reconstruction were performed successfully. The results of follow-up for 72 months showed that there were no signs of recurrence as local or distant metastasis. This case of local recurrence in the IVC highlights that active long-term surveillance for RCC patients of all stages is important for the early diagnosis of tumor recurrence, which improves the potential resectability.
\end{abstract}

\section{Introduction}

Renal cell carcinoma (RCC) has the highest mortality rate of the genitourinary cancers, and accounts for $2 \%$ of all cancers in the world. In China, RCC is the second most frequent genitourinary malignancy, with a steady increase in the incidence in recent decades. One-third of patients are initially diagnosed with disease that is locally invasive or that has already reached stage IV. The only potentially effective treatment for primary and metastatic RCC is surgical resection (1), although recurrence occurs in $\sim 25 \%$ of patients following nephrectomy (2).

Correspondence to: Professor Dexin Yu, Department of Urology, Second Affiliated Hospital of Anhui Medical University, 678 Furong Road, Hefei, Anhui 230022, P.R. China

E-mail: yudx_urology@126.com

Key words: recurrence, inferior vena cava, renal cell carcinoma, radical nephrectomy
The disease remains one of the most treatment-resistant malignancies and is associated with a poor prognosis. It has previously been found that the prognosis for patients with distant disease is generally poor without systemic therapy, including immunotherapy, radiation and targeted treatment, with a 5 -year survival rate of $\leq 10 \%$ (1).

$\mathrm{RCC}$ is unique in that it has the propensity for vascular invasion, namely invasion into the renal vein, and subsequently the inferior vena cava (IVC) or intracardially (3), forming a tumor thrombus. However, the isolated recurrence of RCC in IVC following surgical resection is a rare event. A previous study indicated that the recurrence can happen locally, with an incidence of $1.61 \%$ following surgery (4). The resection of a recurrent tumor thrombus and reconstruction of the IVC often makes clinical management challenging. However, aggressive surgical management in patients with tumor recurrence may offer an opportunity for cure or palliation. The current study presents our experience in the surgical management of a patient with an isolated local recurrence of RCC in the IVC following radical nephrectomy.

\section{Case report}

Radical nephrectomy. A 59-year-old male was admitted to the Department of Urology, First Affiliated Hospital of Anhui Medical University, on February 25, 2003, due to a 3-month history of severe gross hematuria. The patient had a medical history of hypertension controlled by oral medication for 3 years. The patient's body weight and height were $80 \mathrm{~kg}$ and $172 \mathrm{~cm}$, respectively, with a body mass index of 27.04. Vital signs were stable following a physical examination, and no superficial lymph nodes were found. The results from an electrocardiogram, pulmonary function test, stool analysis and other routine laboratory tests were all within normal limits, with the exception of the level of fibrinogen $[5.2 \mathrm{~g} / \mathrm{l}$ (normal range, $\sim 2.0-4.0 \mathrm{~g} / 1)]$. Computed tomography (CT) of the abdomen revealed an enhancing well-defined heterogeneous large mass $(6.5 \mathrm{~cm}$ in maximal diameter) in the lower pole of the right kidney and extending through Gerota's fascia. No para-aortic lymph nodes or lung metastatic lesions were detected by CT scan. Magnetic resonance imaging (MRI) showed no evidence of renal vein or caval thrombi or emboli.

The patient underwent a radical nephrectomy, including lymphadenectomy. Histopathology of the surgical specimen 
diagnosed a grade 2 clear cell carcinoma, with a negative surgical margin. The pathological stage of the tumor was pT3aNOM0.

Surgical management of the recurrent tumor. On January 5, 2007, 4 years after the radical nephrectomy, the patient presented to the Department of Urology, First Affiliated Hospital of Anhui Medical University, with bilateral lower extremity edema. Upon analysis with Doppler ultrasonography, a hypoechoic mass, $3.86 \times 3.70 \mathrm{~cm}$ in size, was located in the IVC. MRI clearly showed a tumor thrombus within the IVC, $4.10 \times 2.80 \mathrm{~cm}$ in size, extending from the infrarenal level to the level of the left renal vessels, with no adherence within the vena cava (Fig. 1).

Under general anesthesia, a chevron incision provided excellent exposure of the IVC, the left kidney and the liver. A thrombectomy and partial IVC resection were performed, and the defect was reconstructed with a polytetrafluoroethylene (PTFE) prosthetic graft. In order to prevent potential hemodynamic instability, the superior and IVC were clamped to the tumor thrombus during the surgery. Initially, am attempt was made to remove the thrombus down to the level of the left renal vessels, however, this was not successful, as adherence of the thrombus with the vena caval wall was subsequently observed. Therefore, the tumor thrombus and the wall of the IVC $(\sim 7 \mathrm{~cm}$ in length) were excised, and reconstruction of the IVC was performed using a PTFE prosthetic graft (Fig. 2). The surgical duration was $\sim 4 \mathrm{~h}$. The total amount of blood loss was $\sim 600 \mathrm{ml}$ and the amount of blood transfused was $\sim 400 \mathrm{ml}$. The patient was discharged home without severe complications after an 11-day hospital stay.

Macroscopic evaluation of the specimen showed that the tumor thrombus was $4.0 \times 3.0 \times 1.5 \mathrm{~cm}$ in size. Histopathological examination demonstrated that the thrombus consisted of clear cancer cells. The pathological diagnosis was of clear cell RCC (Fig. 3), Fuhrman grade 2. Therefore, the overall diagnosis of the patient was the local recurrence of RCC in the IVC with caval wall infiltration. Adjuvant interferon therapy was performed in the post-operative course (Interferon, $3 \mathrm{MIU} / \mathrm{iH}$, 3 times/week for a duration of 12 weeks). The patient was in good general health; proteinuria was observed 3 years post-surgery, however, further treatment was not required. Routine follow-up has demonstrated no signs of reoccurrence as local or distant metastasis for 72 months.

\section{Discussion}

To the best of our knowledge, RCC has a biological behavioral tendency for venous system invasion, with extension into the renal vein $(50 \%)$, IVC $(4-10 \%)$ or right atrium $(\sim 1 \%)$ of new cases diagnosed as RCC $(5,6)$. Although local recurrence following radical nephrectomy is another biological behavior, which may recur at numerous locations and occurs in 2-3\% of patients (7), the late local recurrence of an RCC thrombus extending from the IVC is rare; a thorough review of the published English literature revealed $<10$ cases, including the present study (8-12). In the present study, a patient with local recurrence of a tumor thrombus extending from the IVC 4 years after a right radical nephrectomy for advanced RCC is reported.

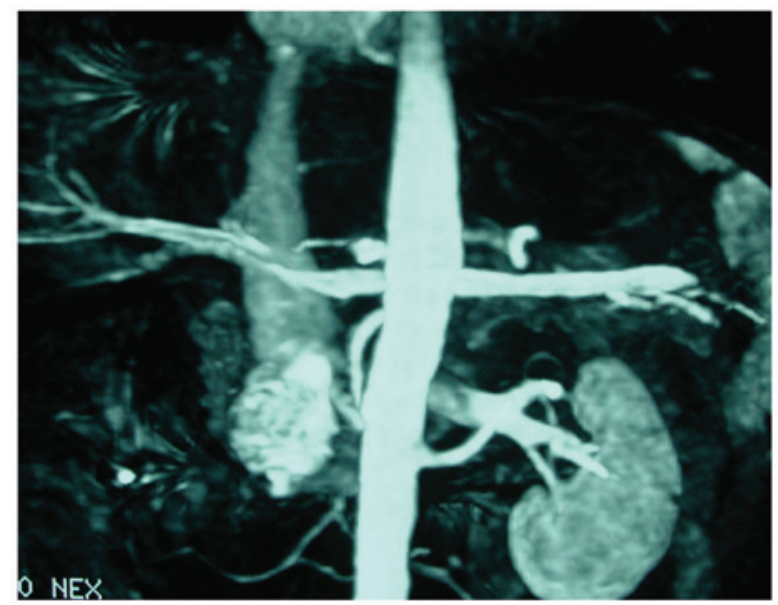

Figure 1. Magnetic resonance imaging of the tumor thrombus, measuring $4.10 \times 2.80 \mathrm{~cm}$, located in the inferior vena cava.

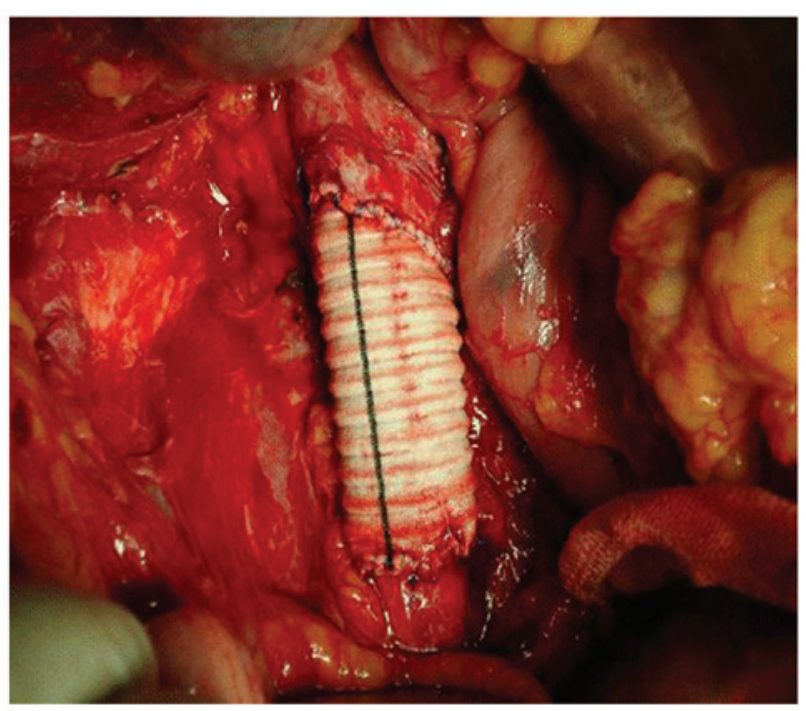

Figure 2. Reconstruction of the inferior vena cava (IVC) during the surgery. The tumor thrombus and part of the IVC wall were surgically removed, and a polytetrafluoroethylene prosthetic graft was used to replace the wall defect.

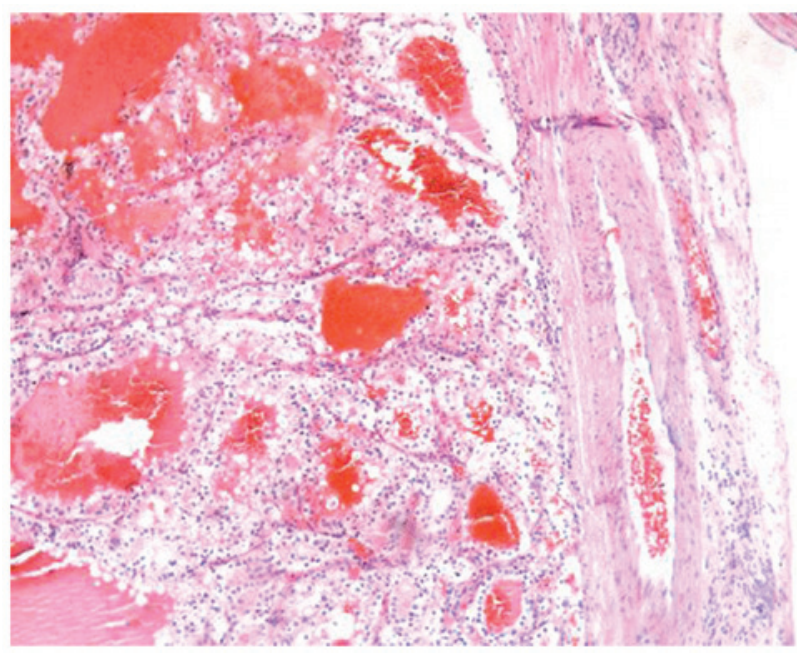

Figure 3. Hematoxylin and eosin staining of the tumor thrombus showing clear cancer cells in the tumor thrombus on histopathological examination. The pathological diagnosis was of clear cell renal cell carcinoma (magnification, x100). 
From the recorded cases, patients with RCC extending into the IVC primarily presented with clinical signs or symptoms of venous obstruction (1), such as dilated superficial abdominal wall veins, caput medusae, pulmonary embolus or proteinuria $(8,9)$. Owing to a slow-growing tumor thrombus and progressive IVC obstruction, up to $40 \%$ of patients reported were asymptomatic. However, the symptoms of venous obstruction appeared in another $40 \%$ of reported cases. In the present case, the patient exhibited significant lower extremity edema.

In total, $>30 \%$ of patients with non-metastatic RCC developed local or systemic tumor recurrence following a radical surgery (13). The recurrent tumor appeared in nearly all organs of the body, but most commonly in the lungs, bone, brain and liver, although IVC recurred rarely. Surgical treatment for recurrent patients who have no signs of local or distant metastasis is considered to be beneficial. Systemic adjuvant medical therapy conferred certain benefits in managing the patients with distant metastasis, however, appeared to be of limited benefit in cases of local recurrence (7). In the present case, the patient underwent successful surgical resection of the tumor thrombus and part of the IVC, with defect reconstruction of the IVC. There were no local or distant metastasis signs within 6 years of follow-up. The results indicated that radical surgery may be a potential treatment for the local recurrence of RCC.

The long-term survival of patients with locally recurrent RCC is poor, with a 5-year survival rate of $28 \%$ (7). The early detection of tumor recurrence can provide the best chance for the long-term survival of patients with RCC. Risk factors associated with the local recurrence of RCC include the size of the primary tumor, a high-grade, invasion of the IVC and involvement of regional lymph nodes (14). The reasons for local recurrence in the IVC may be similar. However, a reasonable explanation for local recurrence in the IVC is unclear. Associated studies have indicated that local recurrence may be associated with the involvement of the IVC wall, tumor seeding $(8,9)$ or skip metastasis, which had occurred prior to radical nephrectomy. Moreover, the right renal vein is shorter than the left side, which may be more convenient for the migration of tumor cells. A review of the literature and the present case indicated ten published cases of RCC recurring in the IVC with no local recurrence or distant metastases, 9 right and 1 left. Therefore, $90 \%$ of local recurrences in reported cases were of right RCC (8-12). In the present case, the tumor recurrence in the IVC was confirmed in the patient following radical nephrectomy for right $\mathrm{RCC}$. At a 72-month follow-up with strict surveillance strategies following the second surgery on the tumor recurrence, there were no signs of recurrence as local or distant metastasis.

The prognostic impact of the level of the tumor thrombus is controversial. One study reported that the level of tumor thrombus was an independent prognostic factor for survival. Lambert et al reported that the survival rate decreased with a higher level of tumor thrombus (15). By contrast, other studies proposed that the presence of a tumor thrombus did not reduce the survival rate and increase the risk of further metastasis $(5,16,17)$. IVC invasion and a friable thrombus were reported as independent prognostic factors in two studies $(18,19)$. In general, non-metastatic renal tumors with venous thrombi have a better prognosis following nephrectomy and tumor thrombectomy compared with patients with metastatic disease. Unfortunately, the presence of a thrombus is often associated with a larger tumor and lymph node or distant metastases, which means a poor prognosis for patients (1). However, there are limited studies on the recurrence of RCC extending from the IVC, thus there is little available information with regard to the treatment and prognosis.

To the urologist, the treatment of the local recurrence of $\mathrm{RCC}$ in the IVC is the real challenge. At present, thrombectomy with a partial IVC resection is the potential treatment for the local recurrence of RCC in the IVC. The surgical approach for the removal of a caval thrombus depends on the level of the thrombus and the adherence with the vena cava (19). Therefore, accurate determination of the extent of IVC involvement is crucial. Although a number of techniques can be used to assess the presence and level of the tumor thrombus, such as abdominal ultrasonography, CT and MRI, at present, MRI is the gold standard for assessing the level of an IVC thrombus $(20,21)$. MRI provides better delineation of the association of the caval wall and the tumor embolus (22).

It is widely agreed that open surgery is essential for the removal of a thrombus that extends into the IVC or atrium. The use of hand-assisted and pure laparoscopic radical nephrectomy have been reported, which may be safe and effective $(23,24)$, although the methods can only be applied in selected cases with low level thrombi, and require high levels of experience and skill. However, no such studies have been reported for recurrence of an RCC thrombus. If a tumor thrombus invades the wall of the cava, resection of the caval wall is inevitable, which ensures negative surgical margins and reduces the risk of late recurrence from the venous wall $(11,25)$. Controversy remains with regard to the reconstruction of the IVC. A previous study proposed that the reconstruction of the IVC may damage the venous collaterals and reduce the collateral venous return (26). In the present case, owing to the adherence of the tumor thrombus to the venous wall and the partial IVC resection, it was necessary to reconstruct the IVC with a PTFE prosthetic graft.

In order to diagnose tumor recurrence early, intensive surveillance plays a key role in all tumor patients even when complete surgical resection of all tumors has been performed. No firm conclusions can be reached from the limited and tenuous information available, although the present study suggests that IVC thrombectomy may be the only potential treatment for the local recurrence of RCC in the IVC. Further studies are required for the management of $\mathrm{RCC}$ recurrence and progression in the IVC.

\section{Acknowledgements}

This study was supported by the Project of Cultivate Scientific Research Foundation (grant no. F1407D).

\section{References}

1. Rini BI, Campbell SC and Escudier B: Renal cell carcinoma. Lancet 373: 1119-1132, 2009.

2. Patard JJ, Pignot G, Escudier B, et al: ICUD-EAU International Consultation on Kidney Cancer 2010: treatment of metastatic disease. Eur Urol 60: 684-690, 2011.

3. Karnes RJ and Blute ML: Surgery insight: management of renal cell carcinoma with associated inferior vena cava thrombus. Nat Clin Pract Urol 5: 329-339, 2008. 
4. Chow JJ, Ahmed K, Fazili Z, Sheikh M and Sheriff M: Solitary renal fossa recurrence of renal cell carcinoma after nephrectomy. Rev Urol 16: 76-82, 2014

5. Manassero F, Mogorovich A, Di Paola G, et al: Renal cell carcinoma with caval involvement: contemporary strategies of surgical treatment. Urol Oncol 29: 745-750, 2011.

6. Kim HL, Zisman A, Han KR, et al: Prognostic significance of venous thrombus in renal cell carcinoma. Are renal vein and inferior vena cava involvement different? J Urol 171: 588-591, 2004.

7. Itano NB, Blute Ml, Spotts B and Zincke H: Outcome of isolated renal cell carcinoma fossa recurrence after nephrectomy. J Urol 164: 322-325, 2000.

8. Finkelstein MP, Drinis S, Tortorelis DG, et al: Recurrence of renal cell carcinoma with extensive vena caval thrombus three years after radical nephrectomy. Urol Int 68: 199-201, 2002.

9. Minervini A, Salinitri G, Lera J, et al: Solitary floating vena caval thrombus as a late recurrence of renal cell carcinoma. Int J Urol 11: 239-242, 2004.

10. Horger DC, Bissada NK, Curry NS and Chaudhary UB: Isolated late recurrence of renal cell carcinoma in the inferior vena cava. Can J Urol 11: 2467-2469, 2004.

11. Smaldone MC, Cannon GM Jr and Hrebinko RL: Resection of recurrent inferior vena cava tumor after radical nephrectomy for renal cell carcinoma. Urology 67: 1084.e5-1084.e7, 2006.

12. Smith RB: Long-term survival of a vena caval recurrence of renal cell carcinoma. J Urol 125: 575-578, 1981.

13. Zisman A, Pantuck AJ, Wieder J, et al: Risk group assessment and clinical outcome algorithm to predict the natural history of patients with surgically resected renal cell carcinoma. J Clin Oncol 20: 4559-4566, 2002.

14. Klatte T, Lam JS, Shuch B, et al: Surveillance for renal cell carcinoma: why and how? When and how often? Urol Oncol 26 550-554, 2008

15. Lambert EH, Pierorazio PM, Shabsigh A, et al: Prognostic risk stratification and clinical outcomes in patients undergoing surgical treatment for renal cell carcinoma with vascular tumor thrombus. Urology 69: 1054-1058, 2007.
16. Sweeney P, Wood CG, Pisters LL, et al: Surgical management of renal cell carcinoma associated with complex inferior vena caval thrombi. Urol Oncol 21: 327-333, 2003.

17. Tanaka M, Fujimoto K, Okajima E, et al: Prognostic factors of renal cell carcinoma with extension into inferior vena cava. Int J Urol 15: 394-398, 2008.

18. Ljungberg B, Stenling R, Osterdahl B, et al: Vein invasion in renal cell carcinoma: Impact on metastatic behavior and survival. J Urol 154: 1681-1684, 1995.

19. Bertini R, Roscigno M, Freschi M, et al: Impact of venous tumour thrombus consistency (solid vs friable) on cancer-specific survival in patients with renal cell carcinoma. Eur Urol 60: 358-365, 2011

20. Kallman DA, King BF, Hattery RR, et al: Renal vein and inferior vena tumor thrombus in renal cell carcinoma: CT, US, MRI and venacavography. J Comput Assist Tomogr 16: 240-247, 1992.

21. Kirkali Z and Van Poppel H: A critical analysis of surgery for kidney cancer with vena cava invasion. Eur Urol 52: 658-662, 2007.

22. Pouliot F, Shuch B, Larochelle JC, et al: Contemporary management of renal tumors with venous tumor thrombus. J Urol 184: 833-841, 2010.

23. Guzzo TJ, Schaeffer EM, McNeil BK, et al: Laparoscopic radical nephrectomy for patients with pathologic T3b renal-cell carcinoma: the Johns Hopkins experience. J Endourol 23: 63-67, 2009.

24. Henderson A, Murphy D, Jaganathan K, et al: Hand-assisted laparoscopic nephrectomy for renal cell cancer with renal vein tumor thrombus. Urology 72: 268-272, 2008.

25. Hardwigsen J, Baqué P, Crespy B, et al: Resection of the inferior vena cava for neoplasms with or without prosthetic replacement: a 14-patient series. Ann Surg 233: 242-249, 2001.

26. Sarkar R, Eilber FR, Gelabert HA, et al: Prosthetic replacement of the inferior vena cava for malignancy. J Vasc Surg 28: 75-83, 1998. 